\title{
AUTOMORPHISMS OF SURFACES IN A CLASS OF WEHLER K3 SURFACES WITH PICARD NUMBER 4
}

\author{
ARTHUR BARAGAR
}

\begin{abstract}
In this paper, we find the group of automorphisms (up to finite index) for $\mathrm{K} 3$ surfaces in a class of Wehler K3 surfaces with Picard number 4. In doing so, we demonstrate a variety of techniques, both general and ad hoc, that can be used to find the group of automorphisms of a K3 surface, particularly those with small Picard number.
\end{abstract}

Introduction. Given an algebraic K3 surface $\mathcal{X}$ defined over a number field $k$, what is its group of automorphisms $\mathcal{A}=\operatorname{Aut}(\mathcal{X} / k)$ ? In this paper, we offer some ideas of how to answer this natural question, and demonstrate these ideas by applying them to a particular example. This paper grew out of a talk given at the Banff International Research Station in December 2008.

There are three main general tools. (1) Every automorphism $\sigma$ induces a linear action on the Picard lattice $\operatorname{Pic}(\mathcal{X})$ that preserves the intersection pairing; (2) the intersection pairing on the Picard lattice is a Lorentz product, so induces a hyperbolic structure on $\mathbb{H}=\mathcal{L}^{+} / \mathbb{R}^{+}$, where $\mathcal{L}^{+}$is the light cone; and (3) a fundamental result due to Pjateckii-S̆apiro and S̆afarevič, which establishes a correspondence between $\mathcal{A}$ and a particular subgroup of the lattice preserving isometries of $\mathbb{H}$.

We apply these results, together with some ad hoc results, to a class of K3 surfaces, and come up with a group of finite index in $\mathcal{A}$. Though not complete, we consider this answer to be sufficient; completing the problem likely depends on the arithmetic and not the geometry, i.e., it depends on $\mathcal{X}$ and not just on $\operatorname{Pic}(\mathcal{X})$.

2010 AMS Mathematics subject classification. Primary 14J27, 14J28, 14J50.

Keywords and phrases. K3 surface, automorphism, Hausdorff dimension, ample cone.

The author was supported by the National Security Agency under grant No. H98230-08-1-0022.

Received by the editors on July 13, 2013, and in revised form on March 18, 2014. DOI:10.1216/RMJ-2016-46-2-399 Copyright (C)2016 Rocky Mountain Mathematics Consortium 
It is clear to the author that these techniques are applicable to many classes of K3 surfaces, particularly those with small Picard number. The hyperbolic space $\mathbb{H}$ of a surface with Picard number $n$ is $n-1$ dimensional, hence the difficulty of dealing with surfaces with large Picard number includes our difficulty imagining hyperbolic spaces of large dimension.

1. Background. Let $\mathcal{X} / k$ be a $\mathrm{K} 3$ surface defined over a number field $k$. Let $n$ be the dimension of the Picard lattice $\operatorname{Pic}(\mathcal{X})$, and let $\left\{D_{1}, \ldots, D_{n}\right\}$ be a basis over $\mathbb{Z}$, so

$$
\operatorname{Pic}(\mathcal{X})=D_{1} \mathbb{Z} \oplus \cdots \oplus D_{n} \mathbb{Z} .
$$

Let $J=\left[D_{i} \cdot D_{j}\right]$ be the intersection matrix for the basis $\mathcal{D}$. By the Hodge index theorem, $J$ has signature $(1, n-1)$, i.e., it has one positive eigenvalue and $n-1$ negative eigenvalues. It therefore defines a Lorentz product, so $\operatorname{Pic}(\mathcal{X}) \otimes \mathbb{R}$ is a Lorentz space. Let $D$ be an ample divisor in $\operatorname{Pic}(\mathcal{X})$. We define the light cone to be the set

$$
\mathcal{L}^{+}=\{x \in \operatorname{Pic}(\mathcal{X}) \otimes \mathbb{R}: x \cdot x>0, x \cdot D>0\} .
$$

The space $\mathbb{H}=\mathcal{L}^{+} / \mathbb{R}^{+}$, together with the distance $|A B|$ defined by

$$
\|A\|\|B\| \cosh |A B|=A \cdot B,
$$

is an $n-1$ dimensional hyperbolic geometry. (By $\|x\|$, we mean $\sqrt{x \cdot x}$.)

An automorphism $\sigma \in \mathcal{A}=\operatorname{Aut}(\mathcal{X} / k)$ acts linearly on the Picard lattice via the pull back map $\sigma^{*}$. We are therefore interested in the linear automorphisms of the Picard lattice, the group

$$
\mathcal{O}=\left\{T \in M_{n \times n}(\mathbb{Z}): T^{t} J T=J\right\} .
$$

In this group is the subgroup of index two that preserves the light cone,

$$
\mathcal{O}^{+}=\left\{T \in \mathcal{O}: T \mathcal{L}^{+}=\mathcal{L}^{+}\right\},
$$

which is a discrete group of isometries on $\mathbb{H}$. It is an arithmetic group, and its fundamental domain has finite volume.

A divisor $E$ is called effective if we can write

$$
E=\sum_{i=1}^{k} a_{i} C_{i},
$$


where $a_{i} \geq 0$ and the $C_{i}$ 's are divisors represented by curves on $\mathcal{X}$. A divisor $D \in \operatorname{Pic}(\mathcal{X}) \otimes \mathbb{R}$ is called ample if $D \cdot E>0$ for all effective divisors $E$. The ample cone $\mathcal{K} \subset \mathcal{L}^{+}$is the set of all ample divisors in $\operatorname{Pic}(\mathcal{X}) \otimes \mathbb{R}$.

Within $\mathcal{O}^{+}$, the reflections through -2 divisors play a special role. Let $C$ be a divisor such that $C \cdot C=-2$. The map $R_{C}$ defined by

$$
R_{C}(\mathbf{x})=\mathbf{x}+(C \cdot \mathbf{x}) C,
$$

which is a reflection through the hyperplane $C \cdot \mathbf{x}=0$, is an isometry in $\mathcal{O}^{+}$. Let $\mathcal{O}^{\prime}$ be the group generated by all such divisors. Note that, for $T \in \mathcal{O}^{+}$,

$$
R_{T C}=T^{-1} R_{C} T
$$

so

$$
\mathcal{O}^{\prime} \triangleleft \mathcal{O}^{+}
$$

If $\sigma \in \mathcal{A}$ and $E$ is effective, then $\sigma_{*} E=\left(\sigma^{-1}\right)^{*} E$ is effective. Thus, $\sigma^{*} D \cdot E=D \cdot \sigma_{*} E>0$ for all effective $E$ and ample $D$, so $\sigma^{*} D$ is ample. We therefore define

$$
\mathcal{O}^{\prime \prime}=\left\{T \in \mathcal{O}^{+}: T \mathcal{K}=\mathcal{K}\right\},
$$

since the pullback map sends $\mathcal{A}$ into $\mathcal{O}^{\prime \prime}$. Pjateckii- $\breve{-S a p i r o}$ and $\breve{S}$ afarevic prove that the pullback map of $\mathcal{A}$ to $\mathcal{O}^{\prime \prime}$ has finite kernel and cokernel [6], and that $\mathcal{O}^{\prime \prime} \cong \mathcal{O}^{+} / \mathcal{O}^{\prime}$.

The interplay of $\mathcal{A}$ and the groups $\mathcal{O}^{+}, \mathcal{O}^{\prime \prime}$ and $\mathcal{O}^{\prime}$ is quite pretty, as we will see in our example. We note that, given an arbitrary $J$, finding $\mathcal{O}^{+}$is a non-trivial problem.

Remark 1.1. Let $\mathbf{n} \cdot \mathbf{x}=0$ be a hyperplane through the origin in $\operatorname{Pic}(\mathcal{X}) \otimes \mathbb{R}$. Reflection through this hyperplane is given by

$$
R_{\mathbf{n}}(\mathbf{x})=\mathbf{x}-2 \operatorname{proj}_{\mathbf{n}} \mathbf{x}=\mathbf{x}-2 \frac{(\mathbf{x} \cdot \mathbf{n}) \mathbf{n}}{\mathbf{n} \cdot \mathbf{n}} .
$$

Thus, if $\mathbf{n} \in \operatorname{Pic}(\mathcal{X})$ (so has integer entries) and $\mathbf{n} \cdot \mathbf{n}= \pm 1$ or \pm 2 , then $R_{\mathbf{n}} \in \mathcal{O}$. Since the intersection pairings for K3 surfaces are always even (see the adjunction formula), we only ever have $\mathbf{n} \cdot \mathbf{n}= \pm 2$. If $\mathbf{n} \cdot \mathbf{n}=-2$, then the plane intersects $\mathbb{H}$, and we get a reflection through a hyperline in $\mathbb{H}$. These are the reflections through -2 curves mentioned above. If $\mathbf{n} \in \operatorname{Pic}(\mathcal{X})$ and $\mathbf{n} \cdot \mathbf{n}=2$, then the hyperplane does not intersect $\mathbb{H}$ 
and $-R_{\mathbf{n}}(\mathbf{x}) \in \mathcal{O}^{+}$. This is inversion through $\mathbf{n}$, which, when $n=3$, is just rotation by $\pi$ about $\mathbf{n}$. This can be used to find elements of $\mathcal{O}^{+}$.

2. A specific example. Let $\mathcal{X}$ be a surface described by a smooth $(2,2,2)$ form in $\mathbb{P}^{1} \times \mathbb{P}^{1} \times \mathbb{P}^{1}$. Such surfaces are sometimes known as Wehler K3 surfaces, so named since Wehler showed that they are K3 surfaces [8]. Such a surface can be expressed as the zero locus of

$$
F(X, Y, Z)=X_{0}^{2} F_{0}(Y, Z)+X_{0} X_{1} F_{1}(Y, Z)+X_{1}^{2} F_{2}(Y, Z),
$$

where $X=\left(X_{0}, X_{1}\right) \in \mathbb{P}^{1}$, and $F_{i}(Y, Z)$ is a $(2,2)$ form in $\mathbb{P}^{1} \times \mathbb{P}^{1}$ for all $i$. Since a smooth $(2,2)$ form in $\mathbb{P}^{1} \times \mathbb{P}^{1}$ is an elliptic curve, $\mathcal{X}$ is fibered by elliptic curves in each of the three directions.

Let

$$
\begin{aligned}
p_{1}: \mathbb{P}^{1} \times \mathbb{P}^{1} \times \mathbb{P}^{1} & \longrightarrow \mathbb{P}^{1} \\
(X, Y, Z) & \longmapsto X
\end{aligned}
$$

be the projection onto the first component, and similarly define $p_{2}$ and $p_{3}$. Let $D_{i}=p_{i}^{-1} H$ be the pullback of a point $H \in \mathbb{P}^{1}$ for $i=1,2$ and 3 .

A generic surface $\mathcal{X}$ in this class has Picard lattice $D_{1} \mathbb{Z} \oplus D_{2} \mathbb{Z} \oplus D_{3} \mathbb{Z}$ and intersection matrix

$$
J=\left[\begin{array}{lll}
0 & 2 & 2 \\
2 & 0 & 2 \\
2 & 2 & 0
\end{array}\right] .
$$

The generic Wehler K3 surfaces have been studied by Wang [7], Billard [4], and the author [1]. They contain no -2 curves, and their group of automorphisms is well understood [8]. Explicit examples are given in [3].

We will study the class of Wehler K3 surfaces with Picard number 4 and such that $F_{2}(Y, Z)$ factors into linear terms, i.e., $F_{2}(Y, Z)=$ $L_{1}(Y, Z) L_{2}(Y, Z)$ where $L_{i}(Y, Z)$ is a $(1,1)$ form in $\mathbb{P}^{1} \times \mathbb{P}^{1}$. Then $\mathcal{X}$ contains the curve $((0,1), Y, Z)$ such that $L_{1}(Y, Z)=0$, which is rational and hence a -2 curve on $\mathcal{X}$. Let $D_{4}$ be the divisor class for this curve. The surface $\mathcal{X}$ also contains the -2 curve $((0,1), Y, Z)$ such that $L_{2}(Y, Z)=0$, and its divisor is $D_{1}-D_{4}$. It is clear that $D_{1} \cdot D_{4}=0$, and $D_{2} \cdot D_{4}=D_{3} \cdot D_{4}=1$, so $\mathcal{D}=\left\{D_{1}, D_{2}, D_{3}, D_{4}\right\}$ is a 
basis of $\operatorname{Pic}(\mathcal{X}) \otimes \mathbb{R}$ ( since $\mathcal{X}$ has Picard number 4$)$, and in this basis,

$$
J=\left[\begin{array}{cccc}
0 & 2 & 2 & 0 \\
2 & 0 & 2 & 1 \\
2 & 2 & 0 & 1 \\
0 & 1 & 1 & -2
\end{array}\right]
$$

Since the elements of the basis $\mathcal{D}$ are in $\operatorname{Pic}(\mathcal{X})$, the lattice $D_{1} \mathbb{Z} \oplus$ $D_{2} \mathbb{Z} \oplus D_{3} \mathbb{Z} \oplus D_{4} \mathbb{Z}$ is a sublattice of $\operatorname{Pic}(\mathcal{X})$.

Lemma 2.1. The basis $\mathcal{D}$ of $\operatorname{Pic}(\mathcal{X}) \otimes \mathbb{R}$ is a basis of $\operatorname{Pic}(\mathcal{X})$ over $\mathbb{Z}$.

Proof. Suppose that there exists an element $C \in \operatorname{Pic}(\mathcal{X})$ such that $C$ is not in the lattice generated by $\mathcal{D}$. Then there exists a $C^{\prime} \in \operatorname{Pic}(\mathcal{X})$ such that $C^{\prime}$ is in the polytope generated by the elements of $\mathcal{D}$, i.e.,

$$
C^{\prime}=c_{1} D_{1}+c_{2} D_{2}+c_{3} D_{3}+c_{4} D_{4}
$$

with $0 \leq c_{i}<1$. Let $a_{i}=C^{\prime} \cdot D_{i} \in \mathbb{Z}$ and $\mathbf{a}=\left[a_{1}, a_{2}, a_{3}, a_{4}\right]$. Then $\mathbf{a}=J \mathbf{c}\left(\right.$ where $\left.\mathbf{c}=\left[c_{1}, c_{2}, c_{3}, c_{4}\right]\right)$, so $0 \leq a_{1}<4,0 \leq a_{2}<5,0 \leq a_{3}<5$ and $-2<a_{4}<2$. Thus, there are only a finite number of cases to check, which is easily done via computer. We find that the only other possibility for $\operatorname{Pic}(\mathcal{X})$ is the lattice spanned by $\left\{D_{1} / 2, D_{2}, D_{3}, D_{4}\right\}$. Since $D_{1}$ represents an elliptic curve, it cannot be decomposed into elliptic curves, so $\mathcal{D}$ is a basis for $\operatorname{Pic}(\mathcal{X})$ over $\mathbb{Z}$.

Remark 2.2. In the above proof, we appealed to the geometry of the K3 surface. This could not be avoided since, by a result due to Morrison [5], there exists a K3 surface with Picard lattice isomorphic to the lattice spanned by $\left\{D_{1} / 2, D_{2}, D_{3}, D_{4}\right\}$.

3. The automorphisms. Because of its quadratic nature, $\mathcal{X}$ has several obvious automorphisms. Let us fix $Y$ and $Z$, so that $F(X, Y, Z)$ is a quadratic in $X$ with two roots $X$ and (say) $X^{\prime}$. Then the map

$$
\sigma_{1}:(X, Y, Z) \longmapsto\left(X^{\prime}, Y, Z\right)
$$

is an automorphism of $\mathcal{X}$. The pull back $\sigma_{1}^{*}$ has several obvious relations: $\sigma_{1}^{*} D_{2}=D_{2}$ and $\sigma_{1}^{*} D_{3}=D_{3}$ which, together with $\sigma_{1}^{2}=\mathrm{Id}$, 
lead to some obvious intersections, such as:

$$
\sigma_{1}^{*} D_{1} \cdot D_{2}=D_{1} \cdot \sigma_{1 *} D_{2}=D_{1} \cdot \sigma_{1}^{*} D_{2}=D_{1} \cdot D_{2}=2 .
$$

The only difficult intersections are $\sigma_{1}^{*} D_{1} \cdot D_{1}=8, \sigma_{1}^{*} D_{1} \cdot D_{4}=4$ and $\sigma_{1}^{*} D_{4} \cdot D_{4}$. Let us fix a curve $C$ in the divisor class $D_{1}$. The image $\sigma_{1} C$ intersects $C$ wherever $F(X, Y, Z)=0$ has a double root, as well as at values of $X$ where both $F=0$ and $\partial_{X} F=0$. This is the intersection of two $(2,2)$ forms, so $\sigma_{1}^{*} D_{1} \cdot D_{1}=8$.

Let us now consider the curve $C$ in $D_{1}$ given by $X=(0,1)$. As $D_{4}$ is a component of $C$, the image $\sigma_{1} C$ intersects $D_{4}$ wherever $X=(0,1)$ is a double root, as well as where $\partial_{X} F=0$ and $L_{1}=0$, which is the intersection of a $(2,2)$ form and a $(1,1)$ form. Thus, $\sigma_{1}^{*} D_{1} \cdot D_{4}=4$. The last intersection is more difficult still, so let us assign it a variable: $\sigma_{1}^{*} D_{4} \cdot D_{4}=a$. Then,

$$
J \sigma_{1}^{*}=\left[\begin{array}{cccc}
8 & 2 & 2 & 4 \\
2 & 0 & 2 & 1 \\
2 & 2 & 0 & 1 \\
4 & 1 & 1 & a
\end{array}\right]
$$

Using $\sigma_{1}^{* 2}=\mathrm{Id}$, we get $a=0$ or 4 , so $\sigma_{1}^{*}=T_{1}$ or $S_{1} T_{1}$, where

$$
T_{1}=\left[\begin{array}{cccc}
-1 & 0 & 0 & -1 \\
2 & 1 & 0 & 1 \\
2 & 0 & 1 & 1 \\
0 & 0 & 0 & 1
\end{array}\right] \text { and } S_{1}=\left[\begin{array}{cccc}
1 & 0 & 0 & 1 \\
0 & 1 & 0 & 0 \\
0 & 0 & 1 & 0 \\
0 & 0 & 0 & -1
\end{array}\right]
$$

Since $S_{1}$ is the isometry that sends $D_{4}$ to $D_{1}-D_{4}$, it is clearly a symmetry of the ample cone $\mathcal{K}$. Thus, both $T_{1}$ and $S_{1} T_{1}$ are in $\mathcal{O}^{\prime \prime}$, and there is no need to resolve the ambiguity for $a$.

In a similar fashion, we can define $\sigma_{2}(X, Y, Z)=\left(X, Y^{\prime}, Z\right)$ and $\sigma_{3}(X, Y, Z)=\left(X, Y, Z^{\prime}\right)$, and their pullbacks $\sigma_{2}^{*}$ and $\sigma_{3}^{*}$. We note that $\sigma_{2}^{*} D_{1}=D_{1}$ and $\sigma_{2}^{*} D_{3}=D_{3}$. As before, the intersection $\sigma_{2}^{*} D_{2} \cdot D_{2}=8$. Looking at the action of $\sigma_{2}$ on the curve $X=(0,1)$, which includes $D_{4}$, we see $\sigma_{2}^{*} D_{4}=D_{1}-D_{4}$, from which we get $\sigma_{2}^{*} D_{2} \cdot D_{4}=D_{2} \cdot\left(D_{1}-D_{4}\right)=1$ and $\sigma_{2}^{*} D_{4} \cdot D_{4}=\left(D_{1}-D_{4}\right) \cdot D_{4}=2$. 
Thus,

$$
J \sigma_{2}^{*}=\left[\begin{array}{cccc}
0 & 2 & 2 & 0 \\
2 & 8 & 2 & 1 \\
2 & 2 & 0 & 1 \\
0 & 1 & 1 & 2
\end{array}\right], \quad \text { so } \quad \sigma_{2}^{*}=\left[\begin{array}{cccc}
1 & 2 & 0 & 1 \\
0 & -1 & 0 & 0 \\
0 & 2 & 1 & 0 \\
0 & 0 & 0 & -1
\end{array}\right]
$$

By symmetry, $\sigma_{3}^{*}=S_{2} \sigma_{2}^{*} S_{2}$ where

$$
S_{2}=\left[\begin{array}{llll}
1 & 0 & 0 & 0 \\
0 & 0 & 1 & 0 \\
0 & 1 & 0 & 0 \\
0 & 0 & 0 & 1
\end{array}\right]
$$

To see whether we have the full group of automorphisms of $\mathcal{X}$, we turn our attention to the action of $\mathcal{O}^{\prime \prime}$ on $\mathbb{H}$. We can send $\mathbb{H}$ to the Poincaré ball model in the following way. There exists an orthonormal basis with change of basis matrix $Q$ that diagonalizes $J$. Let us write $J=Q^{t} A^{t} J_{0} A Q$, where $J_{0}$ has $(-1,-1,-1,1)$ along the diagonal, $A$ has $\left(a_{1}, a_{2}, a_{3}, a_{4}\right)$ along the diagonal, and $-a_{1}^{2},-a_{2}^{2},-a_{3}^{2}$ and $a_{4}^{2}$ are the eigenvalues of $J$. Then, for a point $P \in \mathcal{L}^{+}$, the point $P^{\prime}=A Q P /\|P\|$ is a point on the surface $x_{1}^{2}+x_{2}^{2}+x_{3}^{2}-x_{4}^{2}=-1$. Let $\pi(P)$ be the stereographic projection of $P^{\prime}$ onto the plane $x_{4}=0$ through the point $(0,0,0,-1)$ (see Figure 1$)$. Then $\pi$ is a map from $\mathbb{H}$ to the Poincaré ball model of hyperbolic geometry.

The Poincaré ball can, in turn, be unfolded into the Poincaré upper half space, using $D_{2}$ as the point at infinity. Note that $D_{2} \cdot D_{2}=0$, so it is on $\partial \mathbb{H}$, the boundary of $\mathbb{H}$. Let $G=\left\langle T_{1}, S_{1}, S_{2}, \sigma_{3}^{*}, R_{D_{4}}\right\rangle \leq \mathcal{O}^{+}$. The eigenspace for $S_{1}$ and the eigenvalue 1 is spanned by $\left\{D_{1}, D_{2}, D_{3}\right\}$, so $S_{1}$ is a reflection in this plane. The map $T_{1}$ is a reflection in a plane that includes $D_{1}$ and $D_{2}$ and is perpendicular to the plane through which $S_{1}$ reflects. That plane includes the point $D_{2}+D_{4}$. The map $S_{2}$ is reflection in a plane that includes $D_{1}$, and it sends $D_{2}$ to $D_{3}$. The plane through which it reflects is therefore represented by a hemisphere whose boundary is a circle through $D_{1}$ that is centered at $D_{3}$. The map $R_{D_{4}}$ is a reflection, it sends $D_{2}$ to $D_{2}+D_{4}$, and it fixes $D_{1}$, so it is a reflection through the hemisphere with boundary a circle centered at $D_{2}+D_{4}$ and through $D_{1}$. This is enough information to sketch the fundamental domain for $G$, which is shown in Figure 2. Though our sketch does not need to be too precise, we note that the angle between 


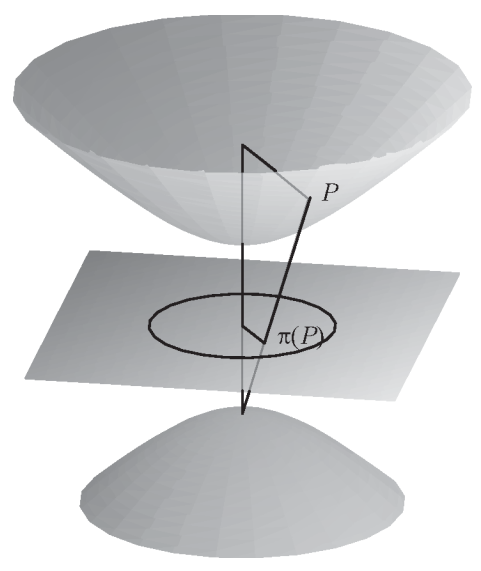

Figure 1. The projection of $\mathbb{H}$ to the Poincaré ball.

the circle that represents $R_{D_{4}}$ and the line that represents $T_{1}$ is $\pi / 4$. To see this, note that the normal $\mathbf{n}_{1}$ to the plane through which $T_{1}$ reflects is $\mathbf{n}_{1}=-D_{1}+D_{2}+D_{3}$ (the eigenvector of $T_{1}$ associated to -1 ), and that

$$
\frac{D_{4} \cdot \mathbf{n}_{1}}{\left\|D_{4}\right\|\left\|\mathbf{n}_{1}\right\|}=\frac{\sqrt{2}}{2}=\cos (\pi / 4)
$$

As can be seen, the fundamental domain has infinite volume. Since the fundamental domain of $\mathcal{O}^{+}$has finite volume, we know we are missing something.

To find another automorphism, we look at the second column of $J$, which is $\left[D_{2} \cdot D_{i}\right]=[2,0,2,1]$. Recall that $D_{2}$ is the divisor class given by the fibers over fixed $Y$, which are generically elliptic curves. Note that $D_{2} \cdot D_{4}=1$, so the rational curve represented by $D_{4}$ intersects each of these elliptic curves exactly once, i.e., we have a fibration of elliptic curves with section. Let $E$ be an elliptic curve in $D_{2}$, and let $O_{E}=E \cap D_{4}$ be its zero element.

We define a map $\sigma_{4}$ on $\mathcal{X}$ in the following way: For $P \in \mathcal{X}$, let $E$ be the unique elliptic curve in $D_{2}$ that contains $P$. Define $\sigma_{4}(P)=-P$, using the group law on $E$ with zero element $O_{E}$. Then $\sigma_{4}$ is an automorphism of $\mathcal{X}$. Since $\sigma_{4}(E)=E$, we know $\sigma_{2}^{*} D_{2}=D_{2}$ and, since $\sigma_{4}\left(O_{E}\right)=O_{E}$, we get $\sigma_{4}^{*} D_{4}=D_{4}$. We also note that $\sigma_{4}^{2}$ is the 


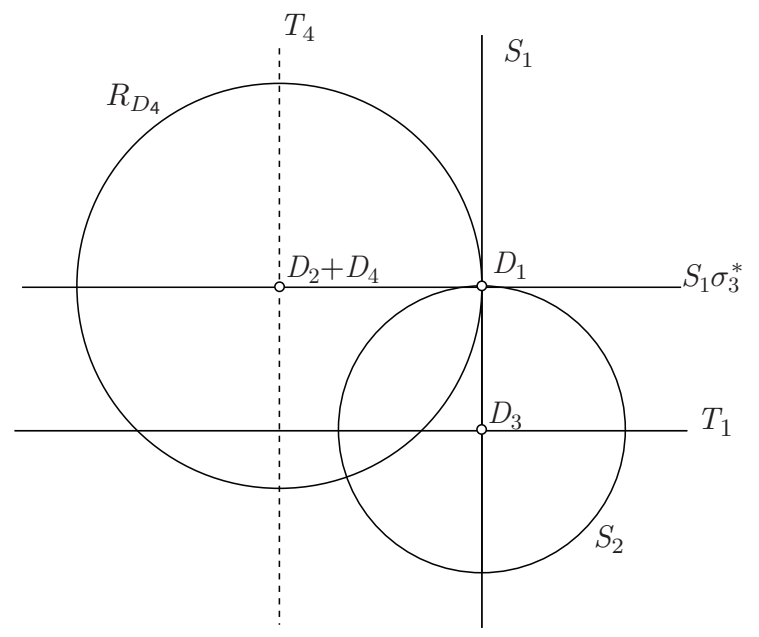

FIGURE 2. The upper half-space representation of isometries in $\mathcal{O}^{+}$. Each line or circle represents the plane or hemisphere above it and is labeled with the isometry that is a reflection through that (hyperbolic) plane. A fundamental domain for $G$ is the region above the two hemispheres and bounded by the planes represented by the solid lines. This region has infinite volume. The fundamental domain for $G^{+}$is the region above the hemispheres and bounded by the four planes represented by the three solid lines and the dotted line. This region has finite volume.

identity. Thus, $\sigma_{4}^{*}$ is either the identity, rotation by $\pi$ about the line with endpoints $D_{2}$ and $D_{2}+D_{4}$, or reflection through a plane that includes that line.

Though there are infinitely many reflections that include a given line, there are significant limitations on what they can be. Let $R_{\mathbf{n}}$ be a reflection through the plane $\mathbf{n} \cdot \mathbf{x}=0$. Then $\mathbf{n}$ is an eigenvector of $R_{\mathbf{n}}$ associated to the eigenvalue -1 . Since $R_{\mathbf{n}}$ has integer entries, we may choose $\mathbf{n}$ to have integer entries. Now suppose $R_{\mathbf{m}}$ is another reflection 
in $\mathcal{O}^{+}$. Then the angle $\theta$ between the two planes (if they intersect) is given by

$$
\cos \theta=\frac{ \pm \mathbf{n} \cdot \mathbf{m}}{\|\mathbf{n}\|\|\mathbf{m}\|} .
$$

Thus,

$$
\frac{\cos 2 \theta+1}{2}=\cos ^{2} \theta=\frac{(\mathbf{n} \cdot \mathbf{m})^{2}}{(\mathbf{n} \cdot \mathbf{n})(\mathbf{m} \cdot \mathbf{m})} \in \mathbb{Q} .
$$

The composition of $R_{\mathrm{n}}$ and $R_{\mathrm{m}}$ is a rotation by $2 \theta$ (if the planes intersect), and since $\mathcal{O}^{+}$is an arithmetic group, we know $2 \theta$ is a rational multiple of $\pi$. Since $\operatorname{deg}(\cos 2 \pi / n)=\frac{1}{2} \phi(n)$, the only possibilities for $\cos 2 \theta$ are $0, \pm \frac{1}{2}, \pm 1$.

If $\sigma_{4}^{*}$ is a reflection with normal vector $\mathbf{n}$, then we can solve for $\mathbf{n}$ by noting $\mathbf{n} \cdot D_{2}=0, \mathbf{n} \cdot D_{4}=0$, and using the above argument with the reflection $S_{1}$. We get $\mathbf{n}=D_{1}+D_{2}-D_{3}$ or $\mathbf{n}=D_{1}-4 D_{2}-2 D_{4}$, which gives us $S_{1} \sigma_{3}^{*}$ and

$$
T_{4}=\left[\begin{array}{cccc}
-1 & 0 & -2 & 0 \\
8 & 1 & 8 & 0 \\
0 & 0 & 1 & 0 \\
4 & 0 & 4 & 1
\end{array}\right] .
$$

The rotation by $\pi$ about the line with endpoints $D_{2}$ and $D_{2}+D_{4}$ is the map $S_{1} \sigma_{3}^{*} T_{4}$.

If $\sigma_{4}^{*}=$ Id or $S_{1} \sigma_{3}^{*}$, then it would appear that the discovery of $\sigma_{4}$ has given us nothing new. However, it did lead us to the discovery of $T_{4}$, which is an element of $\mathcal{O}^{+}$. Furthermore, the group $G^{+}=\left\langle T_{1}, S_{1}, S_{2}, \sigma_{3}^{*}, R_{D_{4}}, T_{4}\right\rangle$ has a fundamental domain with finite volume (see Figure 2), so $G^{+}$has finite index in $\mathcal{O}^{+}$. (In fact, $G^{+}=\mathcal{O}^{+}$, since this fundamental domain has no symmetries. We leave the proof to the reader, as the result is not a necessary component of the paper. First we show that such an isometry cannot interchange the cusps $D_{1}$ and $D_{2}$ and then we analyze where the other vertices of the fundamental domain can go.)

If $\sigma_{4}^{*}=T_{4}$ or $S_{1} \sigma_{3}^{*} T_{4}$, then $T_{4} \in \mathcal{O}^{\prime \prime}$. It turns out the converse is also true.

Lemma 3.1. Suppose $T_{4} \in \mathcal{O}^{\prime \prime}$. Then $\sigma_{4}^{*}=T_{4}$ or $S_{1} \sigma_{3}^{*} T_{4}$. 
Proof. Select any five divisors $C_{i}=\left(S_{1} T_{4}\right)^{i} D_{4}$ from the infinite orbit of $D_{4}$ under the action of $\left\langle S_{1} T_{4}\right\rangle$. It is easy to see that this orbit is infinite since $S_{1} T_{4}$ is a translation on the boundary of the upper halfspace. Since $S_{1} T_{4}$ fixes $D_{2}$, we get

$$
C_{i} \cdot D_{2}=\left(S_{1} T_{4}\right)^{i} D_{4} \cdot D_{2}=D_{4} \cdot\left(T_{4} S_{1}\right)^{i} D_{2}=D_{4} \cdot D_{2}=1 .
$$

Note that $S_{1} \sigma_{3}^{*}$ commutes with both $S_{1}$ and $T_{4}$ (they are reflections that are perpendicular to each other), so

$$
S_{1} \sigma_{3}^{*} C_{i}=S_{1} \sigma_{3}^{*}\left(S_{1} T_{4}\right)^{i} D_{4}=\left(S_{1} T_{4}\right)^{i}\left(S_{1} \sigma_{3}^{*}\right) D_{4}=\left(S_{1} T_{4}\right)^{i} D_{4}=C_{i} .
$$

And, of course, the image of $C_{i}$ under the identity is also $C_{i}$.

Finally, since $T_{4} \in O^{\prime \prime}$, each of the divisors $C_{i}$ are nodal, i.e., they each represent a rational curve. Let $E$ be an elliptic curve in $D_{2}$ that does not include any of the finite number of intersections given by the five $C_{i}$ 's taken in pairs, and let $P_{i}=C_{i} \cap E$, where we are now using $C_{i}$ to represent both a divisor class and (in this usage) the unique curve in that divisor class. Then, of course, $\sigma_{4}\left(P_{i}\right)=-P_{i}$. On the other hand, $\sigma_{4}\left(P_{i}\right)=\sigma_{4}\left(C_{i} \cap E\right)$, so is in $\sigma_{4}^{*} C_{i} \cap E$. But $\sigma_{4}^{*} C_{i}=C_{i}$, and since $C_{i} \cdot D_{2}=1$, there is only one point in this image, namely, $P_{i}$, i.e., $-P_{i}=\sigma_{4}\left(P_{i}\right)=P_{i}$ so $2 P_{i}=0$. But $E$ has at most four points $P$ such that $2 P=0$, giving us a contradiction. Thus, $\sigma_{4}^{*}$ cannot be the identity or $S_{1} \sigma_{3}^{*}$, so it must be either $T_{4}$ or $S_{1} \sigma_{3}^{*} T_{4}$.

We, therefore, have an incentive to prove $T_{4}$ is in $\mathcal{O}^{\prime \prime}$.

The groups $\mathcal{O}^{\prime}$ and $\mathcal{O}^{\prime \prime}$ intersect at just the identity. Thus, an element $T \in \mathcal{O}^{+}$is in $\mathcal{O}^{\prime \prime}$ if and only if $T D$ is ample for any (and all) ample $D$. This gives us an incentive to find more ample divisors.

Lemma 3.2. Suppose $D \in \operatorname{Pic}(\mathcal{X})$ and $C_{0}$ is a nodal curve. Let $Q$ be the perpendicular projection (with respect to the intersection pairing) of $D$ onto the hyperplane $C_{0} \cdot \mathbf{x}=0$. If $D$ is ample, then every point on the open line segment from $D$ to $Q$ is ample. If $D$ is on the boundary of the ample cone, $C_{0} \cdot D \neq 0$, and the line $D Q$ is not in a hyperplane $C \cdot \mathbf{x}=0$ for any nodal curve $C$, then every point on the open line segment from $D$ to $Q$ is ample.

Proof. The ample cone is a polyhedral region bounded by the hyperplanes $C \cdot \mathbf{x}=0$ where $C$ ranges over all nodal curves. If two 
bounding hyperplanes $C_{1} \cdot \mathbf{x}=0$ and $C_{2} \cdot \mathbf{x}=0$ intersect, then the angle $\theta$ of intersection, as measured inside the ample cone, is given by $2 \cos \theta=C_{1} \cdot C_{2}$. Thus, $\theta=\pi / 2$ or $\pi / 3$ (corresponding to $C_{1} \cdot C_{2}=0$ or 1). If not all points between $D$ and $Q$ are ample, then the segment must cross the boundary of the ample cone, i.e., there must be a point $P$ on the line segment such that $P$ is on the hyperplane $C \cdot \mathbf{x}=0$ for some nodal curve $C$. To arrive at a contradiction, we will construct a triangle whose angle sum is greater than $\pi$.

Let $U$ be the subspace spanned by $D, C_{0}$, and $C$, so the intersection of $U$ with $\mathbb{H}$ is a hyperbolic plane. Since $Q$ is in the space spanned by $D$ and $C, Q$ is in $U$. Note that the hyperplanes $C \cdot \mathbf{x}=0$ and $C_{0} \cdot \mathrm{x}=0$ must intersect, for if they do not, then $C_{0} \cdot \mathrm{x}=0$ cannot bound the ample cone anywhere since the ample cone is on the other side of the hyperplane $C \cdot \mathbf{x}=0$. This contradicts $C_{0}$ being nodal. Let $R$ be the point of intersection of $C_{0} \cdot \mathbf{x}=0, C \cdot \mathbf{x}=0, U$, and $\mathbb{H}$. Consider $\triangle P Q R$. Note that $\angle P Q R=\pi / 2$, and $\angle Q R P$ is the supplement of the angle $\theta$ described above, since $P$ is outside the ample cone. Hence, $\angle Q R P \geq \pi / 2$, and the angle sum in $\triangle P Q R$ is greater than $\pi$, a contradiction, so $P$ could not exist.

If $D$ is on the boundary of the ample cone and $C_{0} \cdot D \neq 0$, then $C_{0} \cdot D>0$. If $D+\epsilon Q \notin \mathcal{K}$ for sufficiently small $\epsilon>0$, then there exists a nodal $C$ such that $C \cdot(D+\epsilon Q) \leq 0$ for all $\epsilon>0$ sufficiently small. If the line segment $D Q$ is not on $C \cdot \mathbf{x}=0$, then the inequality is strict. In particular, since $D \in \partial \mathcal{K}$, we have $C \cdot D=0$. If the hyperplanes $C \cdot \mathbf{x}=0$ and $C_{0} \cdot \mathbf{x}=0$ do not intersect, then $C \cdot \mathbf{x}<0$ for all $\mathbf{x}$ such that $C_{0} \cdot \mathbf{x}=0$, i.e., the hyperplane $C_{0} \cdot \mathbf{x}=0$ is not a bounding plane of $\mathcal{K}$, which cannot be the case. Thus, they must intersect and we can construct $R$ as before. If $R \neq Q$, then we arrive at a contradiction as before using the triangle $\triangle D Q R$. If $R=Q$, then the line segment $D Q$ lies on $C^{\prime} \cdot \mathbf{x}=0$.

Thus, $D+\epsilon Q \in \mathcal{K}$ for sufficiently small $\epsilon>0$, and hence the open line segment joining $D+\epsilon Q$ and $Q$ lies in the ample cone. Since $\epsilon>0$ is arbitrary, the open line segment $D Q$ lies in the ample cone.

Since $D_{2}$ represents elliptic curves, it is on the boundary of the ample cone. Since $R_{D_{4}} D_{2}=D_{2}+D_{4}$, the projection of $D_{2}$ onto the plane $D_{4} \cdot \mathbf{x}=0$ has the form $Q=D_{2}+a D_{4}$. Solving for $a$ in $D_{4} \cdot\left(D_{2}+a D_{4}\right)=0$, we get $a=1 / 2$. Thus, $D_{2}+c D_{4}$ is ample 

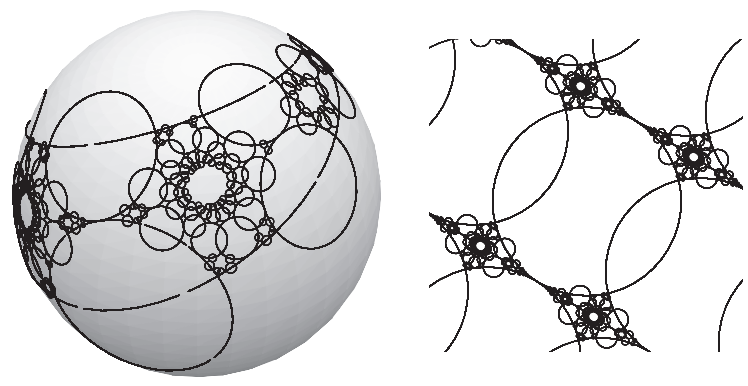

FiguRE 3. The Poincaré ball and upper half-space models of $\mathcal{K} / \mathbb{R}^{+}$. Each circle represents a hyperbolic plane that bounds the region. The region is also a fundamental domain for $\mathcal{O}^{\prime}$.

for $c \in(0,1 / 2)$. In particular, $D_{2}+D_{4} / 4 \in \mathcal{K}$, and since it is in the eigenspace of $T_{4}$ with eigenvalue 1 , we get $T_{4} \in O^{\prime \prime}$. Hence, $\sigma_{4}^{*}=T_{4}$ or $S_{1} \sigma_{3}^{*} T_{4}$.

We therefore conclude $\left\langle\sigma_{1}^{*}, \sigma_{2}^{*}, \sigma_{3}^{*}, \sigma_{4}^{*}\right\rangle$ has finite index (of 1 or 2) in $\mathcal{O}^{\prime \prime}=\left\langle T_{1}, \sigma_{2}^{*}, \sigma_{3}^{*}, \sigma_{4}^{*}, S_{2}\right\rangle=\left\langle T_{1}, \sigma_{2}^{*}, \sigma_{4}^{*}, S_{1}, S_{2}\right\rangle$, and that $\left\langle\sigma_{1}, \sigma_{2}, \sigma_{3}, \sigma_{4}\right\rangle$ has finite index in $\operatorname{Aut}(\mathcal{X})$. We also get

$$
\mathcal{O}^{\prime}=\left\langle R_{T D_{4}}: T \in \mathcal{O}^{\prime \prime}\right\rangle \text {. }
$$

4. The ample cone. The ample cone is the conal region bounded by the planes $C \cdot \mathbf{x}=0$ where $C$ ranges over all nodal curves, i.e., over the $O^{\prime \prime}$ orbit of $D_{4}$. Modulo $\mathbb{R}^{+}$, the ample cone $\mathcal{K}$ lies in $\mathbb{H}$, and is depicted in Figure 3 in the Poincaré ball and upper half-space models. The region $\mathcal{K} / \mathbb{R}^{+}$is also a fundamental domain for $\mathcal{O}^{\prime}$.

Associated to the ample cone is a fractal. Consider the sphere that represents the boundary of $\mathbb{H}$ at infinity in the Poincaré ball model. Every plane that bounds the ample cone $\mathcal{K}$ slices this sphere in two pieces. Remove the piece that represents the half space that does not include $\mathcal{K}$. After doing this for all bounding planes of $\mathcal{K}$, what is left is a fractal. The fractal is also known as the limit set of $\mathcal{O}^{\prime \prime}$ and can be thought of as the set of all points $x \in \partial \mathbb{H}$ such that, for any plane in $\mathbb{H}$ that does not have $x$ on its boundary, and any $P \in \mathbb{H}$, there exists $T \in O^{\prime \prime}$ such that $T(P)$ and $x$ are on the same side of the plane. Experimental calculations suggest the dimension of that fractal 
is $1.415 \pm 0.003$. For a more detailed description of how the calculation is done, see [2].

5. Descent. One often uses a method of descent to navigate through a lattice. Setting up an appropriate height and algorithm for descent is sometimes difficult to accomplish and verify if viewed strictly algebraically. Geometrically, for a group $G=\left\langle R_{\mathbf{n}_{1}}, \ldots, R_{\mathbf{n}_{k}}\right\rangle$ consisting of reflections, the set up is trivially accomplished. Pick any ample divisor $D$ in the interior of the fundamental domain, and for each reflection $R_{\mathbf{n}_{i}}$, verify that $D \cdot \mathbf{n}_{i}>0$, replacing $\mathbf{n}_{i}$ with its negative, if necessary. Define $h(P)=D \cdot P$. For any $P$ not in the closure of the fundamental domain, there exists an $\mathbf{n}_{i}$ such that $\mathbf{n}_{i} \cdot P<0$. We descend by replacing $P$ with $R_{\mathbf{n}_{i}}(P)$, since clearly $h\left(R_{\mathbf{n}_{i}}(P)\right)<h(P)$. Descent ends when no such $\mathbf{n}_{i}$ exists, which means $P$ is in the closure of the fundamental domain.

\section{REFERENCES}

1. A. Baragar, Rational points on K3 surfaces in $\mathbf{P}^{1} \times \mathbf{P}^{1} \times \mathbf{P}^{1}$, Math. Ann. 305 (1996), 541-558.

2. Arthur Baragar, The ample cone for a K3 surface, Canad. J. Math. 63 (2011), 481-499.

3. Arthur Baragar and Ronald van Luijk, K3 surfaces with Picard number three and canonical vector heights, Math. Comp. 76 (2007), 1493-1498 (electronic).

4. Hervé Billard, Propriétés arithmétiques d'une famille de surfaces K3, Comp. Math. 108 (1997), 247-275.

5. D.R. Morrison, On K3 surfaces with large Picard number, Invent. Math. 75 (1984), 105-121.

6. I.I. Pjateckiǔ-Šapiro and I.R. Šafarevič, Torelli's theorem for algebraic surfaces of type K3, Izv. Akad. Nauk 35 (1971), 530-572.

7. Lan Wang, Rational points and canonical heights on K3-surfaces in $\mathbf{P}^{1} \times$ $\mathbf{P}^{1} \times \mathbf{P}^{1}$, Contemp. Math. 186, American Mathematical Society, Providence, RI, 1995.

8. Joachim Wehler, K3-surfaces with Picard number 2, Arch. Math. (Basel) 50 (1988), 73-82.

Department of Mathematical Sciences, University of Nevada, Las Vegas, NV 89154

Email address: baragar@unlv.nevada.edu 